\title{
Análise do impacto de viés nos conjuntos de dados para detecção de Malwares Android
}

\author{
Lucas Vilanova $^{1}$, Renato Sayyed ${ }^{1}$, Taina Soares ${ }^{1}$, \\ Guilherme Siqueira $^{1}$, Gustavo Rodrigues ${ }^{1}$, Eduardo Feitosa ${ }^{2}$, Diego Kreutz ${ }^{1}$ \\ ${ }^{1}$ Universidade Federal do Pampa (Unipampa) \\ ${ }^{2}$ Universidade Federal do Amazonas (UFAM) \\ \{NomeSobrenome\}.aluno@unipampa.edu.br \\ efeitosalicomp.ufam.br, kreutz@unipampa.edu.br
}

\begin{abstract}
Resumo. Atualmente, a detecção de malwares Android é realizada, majoritariamente, através de modelos de aprendizado de máquina. O problema é que a maioria dos modelos desenvolvidos têm sido treinados com conjuntos de dados defasados (e.g., de 2012). Nosso objetivo é coletar evidências iniciais para demonstrar o impacto de diferentes datasets no desempenho de modelos preditivos. Para isto, utilizamos conjuntos de dados de diferentes períodos temporais, isto é, de 2012 a 2021.
\end{abstract}

\section{Introdução}

O sistema Android é atualmente um alvo frequente de aplicações maliciosas (malwares). Estudos apontam que modelos de apredizagem de máquina (machine learning) conseguem detectar malwares com uma acurácia acima de 90\% [Sharma and Rattan, 2021]. Contudo, os malwares aumentam em número e sofisticação, exigindo atualização e desenvolvimento de novos modelos [Sahay et al., 2020].

Como modelos de machine learning aprendem com os dados de entrada, o desempenho (i.e., capacidade preditiva) destes é impactado fortemente pelos datasets (conjunto de dados) utilizados para treino [Allix et al., 2015]. Ainda assim, a maioria das soluções aplicadas à detecção de malwares utiliza datasets antigos (e.g., The Drebin Dataset, de 2012) ou defasados (e.g., características de APIs antigas) [Soares et al., 2021b].

Levantamos a hipótese de que ao treinarmos modelos de machine learning com um dataset defasado, estes não irão apresentar o mesmo poder preditivo para conjuntos de dados atuais. Dentre os diversos indícios que corroboram a hipótese, podemos destacar: a evolução das APIs dos aplicativos Android (da versão 1 em 2008 a versão 31 em 2021); a constante evolução e sofisticação do comportamento dos aplicativos maliciosos; e o aumento expressivo na quantidade de malwares.

Com o objetivo de verificar a hipótese levantada, neste trabalho propomos um experimento para a análise do impacto no desempenho de um modelo de aprendizagem de máquina, a partir de conjuntos de dados de diferentes períodos de tempo (i.e., mais antigos e mais atuais). Para a construção do modelo, escolhemos o algoritmo Random Forest [Breiman, 2001] por ser um dos mais utilizados na detecção de malwares Android [Sharma and Rattan, 2021].

As contribuições do trabalho podem ser resumidas em: (a) caracterização do impacto da utilização de uma mesma configuração de um modelo, adequado a um conjunto 
de dados defasados, em datasets recentes; e (b) identificação de dados duplicados em datasets amplamente utilizados na literatura, bem como demonstração dos efeitos desses dados nos modelos de aprendizado de máquina.

O trabalho está organizado como segue. Nas Seções 2, 3 e 4 apresentamos os datasets, o modelo e os resultados, respectivamente. Na Seção 5 discutimos a atualidade dos dataset e, finalmente, na Seção 6 apresentamos as considerações finais.

\section{Datasets}

Os 4 (quatro) datasets utilizados, selecionados a partir do levantamento apresentado em [Soares et al., 2021b], são apresentados na Tabela 1. Para a seleção dos datasets, utilizamos dois critérios: (1) o mais popular na literatura, naquele ano, e (2) os que estavam publicamente disponíveis. Selecionamos então, conjuntos de dados com o intervalo de 3 anos $(2012,2015,2018$ e 2021).

Tabela 1. Datasets

\begin{tabular}{|c|c|c|c|c|c|c|c|}
\hline \hline \multirow{2}{*}{ Datasets } & \multirow{2}{*}{ Data } & \multicolumn{2}{|c|}{ Com Duplicatas } & \multicolumn{2}{c|}{ Sem Duplicatas } & \multirow{2}{*}{ Permissões } & \multirow{2}{*}{ Permissões após limpeza } \\
\cline { 3 - 7 } & & Benignos & Malignos & Benignos & Malignos & & \\
\hline Drebin-215 & 2012 & 9.476 & 5.560 & 5.539 & 1.720 & 114 & 113 \\
\hline Androcrawl & $2014-2015$ & 139.670 & 23.313 & 138.867 & 23.247 & 61 & 58 \\
\hline AndroidMalwareNormal & 2018 & 18.850 & 9.999 & 16.698 & 7.026 & 173 & 173 \\
\hline DefenseDroid & 2021 & 5.975 & 6000 & 3.146 & 4.579 & 1.490 & 134 \\
\hline \hline
\end{tabular}

Conjuntos de dados como o o Drebin-215 e Androcrawl contêm características como permissões, chamadas de API e intenções. Entretanto, como o objetivo é utilizar apenas permissões, pois são as características mais relevantes e utilizadas [Sharma and Rattan, 2021], removemos as demais características. Ao final do processo de limpeza e seleção de apenas permissões, os datasets Drebin-215, Androcrawl, AndroidMalwareNormal e DefenseDroid ficaram com 113, 58, 173 e 134 permissões, respectivamente.

A preparação dos dados é um conjunto de etapas fundamentais para melhorar e adequar o conjunto de dados ao algoritmo de aprendizado de máquina [Zheng and Casari, 2018]. Por exemplo, a preparação pode evitar ruídos nos dados (e.g., como no caso do Androcrawl dataset, que continha todos os valores com formato textual e apresentava valores inválidos) que poderiam causar erros nos cálculos do modelo por não estarem em um formato numérico ou por apresentarem valores faltantes [Qi et al., 2018]. A preparação dos 4 datasets incluiu a exclusão de características (e.g., a permissão SET_WALLPAPER, duplicada no Drebin-215) de forma a auxiliar o modelo a realizar seus cálculos.

No dataset Androcrawl identificamos três permissões com apenas valores " 0 " (RECEIVE, MESSAGE, SEND) e com 10.262 exemplos de aplicativos Android com valores inválidos (i.e., “?”). Para que o dataset não sofresse uma redução significativa nos 
exemplos de aplicativos caso removêssemos cada registro com valores faltantes, essas três permissões foram removidas.

No DefenseDroid removemos um conjunto de 1.356 features, incluindo duplicatas e permissões que não fazem parte da lista de permissões do Android ${ }^{1}$, resultando em um dataset de 134 permissões. No caso do AndroidMalwareNormal, foram removidas apenas as características "Category" e "Package", que não são permissões.

O que mais nos chamou atenção na etapa de preparação dos dados foram os exemplos de aplicativos Android duplicados. Há 7.777, 5.125, 4.250 e 869 duplicadas nos datasets Drebin-215, AndroidMalwareNormal, DefenseDroid e Androcrawl, respectivamente. Os exemplos duplicados em datasets podem impactar no desempenho dos algoritmos de aprendizado de máquina, podendo levar ao overfitting e enviesamento do modelo [Zhao et al., 2021]. Removemos também todas as duplicatas, mantendo apenas a primeira ocorrência.

Os dados originais, que passaram pelo processo de limpeza e correção, bem como os conjuntos de dados resultantes e suas análises exploratórias, utilizados neste trabalho, estão disponíveis em https : / / github . com/Malware-Hunter/DatasetMatters.

\section{Modelo RandomForest}

O algoritmo RandomForest é baseado em diversas árvores de decisão utilizadas em amostras de dados selecionadas aleatoriamente. A partir das previsões de cada árvore é selecionada a melhor solução por meio de votação.

Os dados de cada um dos 4 datasets foram separados em três partes, sendo $40 \%$ para treino, $30 \%$ para validação e $30 \%$ para teste. Além disso, devido à diferença da amostragem dentre datasets, resolvemos padronizar para o menor conjunto. Isto reduz a probabilidade de que um dataset aprenda mais que outro por conta da quantidade de amostras de aplicativos. Para isso, selecionamos a menor quantidade de cada classe entre todos os datasets ( 3.146 benignos e 1.720 malignos) e a padronizamos como quantidade total de exemplos fornecidos ao conjunto de treino nos 4 conjuntos de dados. Assim, o impacto da diferença da quantidade de exemplos treinados em cada dataset no desempenho do modelo é anulado. Utilizamos o parâmetro random_state $=42$ para selecionar os mesmos conjuntos de dados em cada partição e não modificar os resultados a cada treino.

Como o nosso objetivo é classificar aplicativos entre benignos e malignos, considerando conjuntos de dados desbalanceados, utilizamos a métrica ROC-AUC (ou curva ROC). A curva ROC é uma métrica de avaliação que descreve a capacidade do modelo de distinguir entre duas classes (benignos e malignos), onde é necessário calcular as probabilidades de cada observação (exemplo de um aplicativo) pertencer a uma classe. $\mathrm{O}$ valor de ROC-AUC varia de 0,0 até 1,0, onde o 0,0 representa um modelo que erra todas as predições e 1,0 indica um teste perfeito de predição.

${ }^{1}$ https://developer.android.com/reference/android/Manifest.permission 


\subsection{Otimização do modelo}

O experimento consiste em analisar o desempenho de um modelo, configurado para um dataset defasado, em conjuntos de dados mais recentes. $\mathrm{O}$ dataset de referência selecionado foi o Drebin-215 (de 2012).

Primeiramente, foi necessário balancearmos os dados de treino a fim de nivelar a proporção entre exemplos de aplicações benignas e malignas, pois como a classe majoritária (benignos) está presente em excesso, isso pode ocasionar em overfitting no algoritmo, fazendo com que o modelo acabe errando muitos exemplos da classe minoritária (malignos).

Com o conjunto de dados de treino balanceado, treinamos o classificador RandomForest com seus parâmetros default (i.e., parâmetros padrão fornecidos pela biblioteca do scikit-learn ${ }^{2}$ ) e o validamos com dados desbalanceados para aproximar o teste de um caso real. Com isto, obtivemos um resultado de $89,47 \%$ de ROC-AUC, e com uma garantia maior de não incluir viés no modelo.

O passo seguinte no desenvolvimento de um modelo é ajustar os hiperparâmetros do algoritmo, pois eles impactam nas taxas de detecção [Probst et al., 2019]. Utilizamos a função Randomi zedSearchCV da biblioteca scikit-learn ${ }^{3}$. O espaço de busca dos hiperparâmetros, bem como a combinação ótima de valores encontrada para esse experimento estão descritos na Tabela 2. O resultado do modelo otimizado no dataset baseline Drebin-215, utilizando o treino balanceado, foi de $89,58 \%$ de ROC-AUC.

Tabela 2. Hiperparâmetros do modelo RandomForest

\begin{tabular}{|l|l|l|l|}
\hline \hline Hiperparâmetro & Padrão & Intervalo de busca & RandomizedSearchCV \\
\hline n_estimators & 100 & $200: 2000$ & 1.800 \\
\hline min_samples_split & 2 & 2,5 e 10 & 2 \\
\hline min_samples_leaf & 1 & 1,2 e 4 & 1 \\
\hline max_features & auto & auto e sqrt & auto \\
\hline max_depth & None & $10: 110$ e None & 20 \\
\hline bootstrap & True & True e False & False \\
\hline \hline
\end{tabular}

\section{Resultados}

A partir dos resultados apresentados na Tabela 3, podemos concluir que o modelo configurado para o Drebin-215 obteve resultados muito baixos nos datasets Androcrawl e AndroidMalwareNormal. Isso se deve ao fato desses conjuntos de dados conterem características não relevantes aos cálculos do algoritmo, prejudicando a capacidade preditiva do modelo. Isto, por si só, já corrobora parcialmente a nossa hipótese: é pouco efetivo treinar um modelo com datasets defasados e aplicá-lo, posteriormente, a datasets atuais. $\mathrm{O}$ dataset impacta de forma significativa no desempenho do modelo.

Além disso, podemos observar que as duplicatas também podem causar impacto no desempenho do algoritmo. Elas podem levar o modelo ao enviesamento, pois ao ser

\footnotetext{
${ }^{2}$ https://scikit-learn.org/stable/modules/generated/sklearn. ensemble. RandomForestClassifier.html

${ }^{3}$ https://scikit-learn.org/stable/modules/generated/sklearn.model_ selection.RandomizedSearchCV.html
} 
Tabela 3. Resultados do modelo RandomForest

\begin{tabular}{|c|c|c|}
\hline \hline \multirow{2}{*}{ Dataset } & \multicolumn{2}{|c|}{ ROC-AUC } \\
\cline { 2 - 3 } & Com duplicata & Sem duplicata \\
\hline Drebin-215 (2012) & $94,37 \%$ & $89,58 \%$ \\
\hline Androcrawl (2014/2015) & $55,80 \%$ & $54,39 \%$ \\
\hline AndroidMalwareNormal (2018) & $55,80 \%$ & $50,47 \%$ \\
\hline DefenseDroid (2021) & $84,61 \%$ & $84,85 \%$ \\
\hline \hline
\end{tabular}

testado com um conjunto de dados que contenha exemplos de aplicativos que o mesmo já treinou, ele saberá predizer corretamente [Zhao et al., 2021]. Portanto, eliminar duplicatas é fundamental para treinar um modelo de detecção de malwares.

Na prática, o desempenho do modelo decaiu em 4,79\% para o dataset Drebin-215 com e sem duplicatas. Como o Drebin-215 continha mais de 50\% de exemplos duplicados, o modelo poderia estar enviesado. Com a remoção das duplicatas, temos uma garantia maior de termos um modelo que não sofre de enviesamento.

As duplicatas no dataset AndroidMalwareNormal também impactaram o desempenho do modelo (em 5,33\%). Assim, como no caso do Drebin-215, a redução no resultado, em parte, pode ter sido causada pelo enviesamento e pela redução significativa de exemplos de aplicativos. Já no caso do dataset DefenseDroid, apesar de possuir 35,49\% de dados duplicados, o impacto no resultado do modelo foi insignificante. Isso ocorre pelo fato de uma boa parcela das permissões do dataset serem classificadas como relevantes para a detecção de malwares [Li et al., 2018, Assolin et al., 2021].

\section{Discussão}

Existe uma questão bastante peculiar com relação à atualidade dos dados dos datasets. Inicialmente assumimos que a data do dataset é a mesma da coleta dos dados. Entretanto, durante nossas análises, identificamos que existe uma probabilidade de que os dados sejam mais antigos do que o esperado (e.g., do que o informado no dataset). Esse problema ocorre quando os datasets possuem dados de versões de APIs muito antigas e não possuem nenhum dado de versões das APIs do Android (e.g., versão da API do mesmo ano de criação do dataset ou do ano anterior). Isto pode ocorrer por diferentes razões, como discutimos detalhadamente em outro trabalho [Soares et al., 2021a]. Por exemplo, é comum os autores compilarem datasets a partir de outros datasets previamente existentes (e mais antigos). Consequentemente, as características dos aplicativos (e.g., permissões, chamadas de API) acabam sendo defasadas.

\section{Considerações Finais}

Nesse artigo, avaliamos o impacto de datasets de diferentes datas no desempenho de um modelo baseado no algoritmo RandomForest. O experimento consistiu em treinar e configurar o modelo para o dataset Drebin-215 e avaliar seu desempenho em datasets mais recentes, utilizando todas as permissões presentes nos conjuntos de dados.

Os resultados obtidos indicam que há uma forte relação entre o dataset e o desempenho do modelo. Simplesmente utilizar um dataset defasado não significa que o modelo 
irá performar bem com datasets mais atuais. Primeiro, os datasets podem conter características distintas, o que vai levar o modelo a resultados diferentes do esperado quando este for treinado com dados defasados. Segundo, diferentemente dos datasets defasados, datasets recentes podem incorporar conjuntos de caractetísticas das novas versões das APIs do Android, que não existiam anteriormente, impactando o desempenho dos modelos. Portanto, para detectarmos malwares atuais, é fundamental o treinamento dos novos modelos com dados atuais.

Como trabalhos futuros, podemos destacar: (a) a criação de um dataset com dados atuais, pois os que existem na literatura não contemplam as últimas versões das APIs do Android e não incorporam amostras significativas de aplicativos maliciosos e benignos de 2020 e 2021; (b) investigar extensivamente os enviesamentos causados pelos dados de datasets existentes (e.g., registros duplicatas); e (c) investigar questões relacionadas a rastreabilidade das amostras dos datasets.

\section{Agradecimentos}

Esta pesquisa foi financiada, conforme previsto nos Arts. 21 e 22 do decreto no. 10.521/2020, nos termos da Lei Federal no. 8.387/1991, através do convênio no. 003/2021, firmado entre ICOMP/UFAM, Flextronics da Amazônia Ltda e Motorola Mobility Comércio de Produtos Eletrônicos Ltda.

\section{Referências}

Allix, K., Bissyandé, T. F., Klein, J., and Le Traon, Y. (2015). Are your training datasets yet relevant? In International Symposium on Engineering Secure Software and Systems, pages 51-67. Springer.

Assolin, J., Rocha, V., Kreutz, D., Siqueira, G., Rodrigues, G., Feitosa, E., and Casola, K. (2021). Detecção de Malwares Android: reprodução da seleção de características do SigPID. https: / / arxiv.kreutz.xyz/wrseg2021_sigpid_ve1.pdf.

Breiman, L. (2001). Random forests. Machine learning, 45(1):5-32.

Li, J., Sun, L., Yan, Q., Li, Z., Srisa-An, W., and Ye, H. (2018). Significant permission identification for machine-learning-based android malware detection. IEEE Transactions on Industrial Informatics, 14(7):3216-3225.

Probst, P., Boulesteix, A.-L., and Bischl, B. (2019). Tunability: importance of hyperparameters of machine learning algorithms. The Journal of Machine Learning Research, 20(1):1934-1965.

Qi, Z., Wang, H., Li, J., and Gao, H. (2018). Impacts of dirty data: and experimental evaluation. arXiv preprint arXiv:1803.06071.

Sahay, S. K., Sharma, A., and Rathore, H. (2020). Evolution of malware and its detection techniques. In Information and Communication Technology for Sustainable Development, pages 139-150. Springer.

Sharma, T. and Rattan, D. (2021). Malicious application detection in android-a systematic literature review. Computer Science Review, 40:100373.

Soares, T., Assolin, J., Barcellos, L., Sayyed, R., Casola, K., Kreutz, D., Costa, E., Gustavo, N., and Feitosa, E. (2021a). Detecção de malwares android: Disponibilidade e atualização das fontes de dados. https://arxiv.kreutz.xyz/wrseg2021_disponibilidade_ve1.pdf.

Soares, T., Siqueira, G., Barcellos, L., Sayyed, R., Vargas, L., Rodrigues, G., Assolin, J., Pontes, J., and Kreutz, D. (2021b). Detecção de malwares android: datasets e reprodutibilidade. https: / / arxiv. kreutz.xyz/mh21_reprodutibilidade.pdf.

Zhao, Y., Li, L., Wang, H., Cai, H., Bissyandé, T. F., Klein, J., and Grundy, J. (2021). On the impact of sample duplication in machine-learning-based android malware detection. ACM Transactions on Software Engineering and Methodology (TOSEM), 30(3):1-38.

Zheng, A. and Casari, A. (2018). Feature engineering for machine learning: principles and techniques for data scientists. "O'Reilly Media, Inc.". 